Reprod. Nutr. Dévelop., 1980, 20 (5 B), 1667-1678.

\title{
Intérêt des systèmes gnotoxéniques pour l'étude des relations hôte-flore microbienne du tube digestif
}

\author{
par R. DUCLUZEAU, P. RAIBAUD
}

Laboratoire d'Ecologie Microbienne

I.N.R.A., 78350 Jouy en Josas, France.

Summary. The advantage of gnotoxenic systems in the study of host-microbial flora inferactions in the digestive tract.

It is necessary to use experimental models in all studies of interactions between a host, its digestive tract microbial flora and the environment because these interactions are complex. The gnotoxenic animal, reared in an isolator as the axenic animal and harbouring a known microbial flora, constitutes either an analytic or mimetic experimental model.

The gnotoxenic animal may be considered as an analytic model when used to determine which biotic or abiotic environmental factor of the host-animal plays a role in the intestinal ecosystem. The potential metabolic or immunologic role of a given bacterial strain in the intestinal ecosystem may be determined when the axenic animal is associated with this strain. The variation of the expression of the potential role of this strain in relation to the environment can be ascertained by diversifying the diet of the host or by introducing other bacterial strains into the intestinal ecosystem. The role of an association of strains in host physiology and host protection against potentially pathogenic target strains can also be studied using this analytic model. An analytic model is created by associating either isolated strains of the holoxenic or heated or diluted suspensions of holoxenic digestive tract flora. Axenic mice associated with these simplified flora are called meroxenic.

The gnotoxenic animal is considered to be a mimetic model when it harbours a microbial flora isolated from an animal of a different species. The advantage of the mimetic model is that it provides an easy-to-use biological tool, i.e. gnotoxenic mice, to first determine the potential role of these microbial strains. The validity of the mimetic model is then tested by comparing gnotoxenic mice and gnotoxenic piglets or chickens. When all the gnotoxenic animals are given the same diet, this comparison permits an estimation of the animal-host role in the expression of the potential activities of microbial strains. The mimetic model, composed of gnotoxenic animals harbouring microbial strains of human origin, is the only experimental model which can be used to study the role of microorganisms in the intestinal ecosystem of man.

\section{1. - Introduction.}

Les techniques qui permettent de contrôler complètement l'environnement microbien dans lequel vit un animal ou un homme ont fait de rapides progrès depuis quelques années. Il est maintenant relativement aisé de maintenir un animal soit à l'abri de tout contact avec des populations de microbes vivants (animal axénique), soit associé uniquement à des microbes connus (animal gnotoxénique). Ces animaux maintenus en 
isolateur depuis leur naissance s'opposent à ceux qui naissent et vivent dans un environnement microbien non contrôlé sans précautions spéciales d'asepsie (animaux holoxéniques) ou dans un environnement protégé par certaines barrières physiques qui ne sont pas étanches à tous les microbes (animaux hétéroxéniques).

Parmi les nombreuses améliorations techniques qui ont permis ce progrès, on peut citer l'utilisation d'isolateurs en film de polyvinyl souple, faciles à stériliser, s'adaptant à tous les usages particuliers (Saint-Martin ef al., 1975), l'utilisation de systèmes de transfert rapide (système DPTE) réduisant de $25 \mathrm{~min}$ à quelques secondes la durée des transferts ; l'utilisation de systèmes de pulvérisation automatique de l'antiseptique le plus utilisé, l'acide peracétique, l'utilisation d'aliments commerciaux et de litières préstérilisés par irradiation et faciles à stocker sous cetfe forme (Ducluzeau et al., 1979), la miniaturisation des isolateurs qui permet leur transfert aisé et leur utilisation dans n'importe quel laboratoire sans aménagement spécial (Moreau, Ducluzeau et Meyer, 1978). L'utilisation des animaux gnotoxéniques ef axéniques pourraił certainement être plus répandue dans les laboratoires qu'elle ne l'est aujourd'hui, s'il n'existait encore à l'égard de cette technique une certaine réticence héritée du temps des pionniers où ce type d'élevage relevait en effet de l'exploit technique.

Les techniques de la gnotoxénie ont trouvé des débouchés dans deux domaines différents : celui des applications pour l'élevage des animaux ou la protection de l'homme contre l'environnement microbien el celui de la biologie expérimentale où ces techniques s'ajoutent à celles déjà existantes. On sait maintenant recueillir en conditions axéniques un nouveau-né humain atteint de déficit immunitaire profond et le maintenir axénique jusqu'à ce qu'on ait reconstitué ses défenses immunitaires (Ducluzeau et al., 1975). On sait également utiliser ces techniques pour maintenir à l'abri de toutes les contaminations extérieures des enfants dont le tube digestif est « décontaminé » par des mélanges d'antibiotiques et dont les défenses immunitaires n'ont pas encore été reconstituées (Raibaud ef al., 1975). On prélève en conditions axéniques des porcelets pour les utiliser ensuite comme reproducteurs dans les troupeaux dits Specific Pathogen Free (SPF). Les mêmes techniques permettent d'obtenir des élevages de poulets SPF.

Les animaux axéniques et gnotoxéniques sont aussi utilisés en biologie comme modèles expérimentaux. La gnotoxénie est la branche de la biologie qui utilise ces animaux gnotoxéniques. Le modèle expérimental que constitue l'animal gnotoxénique a une double finalité : lorsque l'on se pose un problème biologique au sujet d'un animal d'intérêt zootechnique, il est parfois impossible d'y apporter une réponse en expérimentant directement sur l'animal concerné pour des raisons techniques (notamment la taille de l'animal) ou économiques (coût des animaux interdisant d'expérimenter sur un nombre suffisant d'individus). Dans le cas de l'homme, cette impossibilité d'expérimenter est évidemment d'ordre éthique. Dans tous ces cas, on cherche à substituer à l'animal concerné ou à l'homme un animal de laboratoire capable de reproduire aussi fidèlement que possible le phénomène biologique exploré : on cherche un modèle expérimental que l'on pourrait qualifier de mimétique. Dans d'autres cas au contraire, on utilise ces modèles expérimentaux à des fins que l'on pourrait qualifier d'analytiques. Le modèle analytique sert à déterminer quel facteur de l'environnement d'un holoxénique joue un rôle dans le phénomène biologique étudié : par exemple, 
quelle population bactérienne du tube digestif agit sur un métabolisme donné, quel composant du régime alimentaire agit sur un élément de la flore. Un animal axénique associé à une ou plusieurs bactéries et recevant un régime alimentaire déterminé représente donc le modèle expérimental analytique. Bien entendu, le modèle ainsi créé est tout à fait artificiel en ce sens que l'on a supprimé les interactions entre le facteur choisi ef les autres facteurs susceptibles de jouer un rôle chez un holoxénique. L'effet de ce facteur s'en trouve souvent amplifié, donc plus facile à mettre en évidence et à étudier, mais il restera à vérifier la signification du résultat obtenu dans une situation écologique plus complexe. Le modèle expérimental analytique sert donc à mettre en évidence les effets biologiques potentiels de telle souche bactérienne, mais aussi à étudier l'expression de cet effet potentiel en fonction de l'environnement. Les deux types de modèles mimétique et analytique ne s'excluent d'ailleurs pas mutuellement : lorsque l'on recherche par exemple l'effet antagoniste à l'égard d'un micro-organisme pathogène de telles bactéries isolées du łube digestif humain en l'ensemençant chez une souris axénique, le modèle expérimental ainsi créé est à la fois mimétique et analytique. Autant que faire se peut d'ailleurs, le modèle analytique doit remplacer le modèle mimétique : ainsi, lorsqu'on veut connaître l'association bactérienne antagoniste d'une bactérie cible chez le porcelet holoxénique, on peut analyser ce phénomène en utilisant d'abord la souris gnotoxénique, modèle mimétique, puis créer un modèle analytique en utilisant des porcelets axéniques.

\section{2. - L'animal gnotoxénique, modèle expérimental analytique.}

\section{1. - Mise en évidence d'un effet biologique potentiel de certaines bactéries.}

De nombreux exemples montrent qu'il est en général impossible de déduire des caractères métaboliques d'une bactérie étudiée in vitro son rôle in vivo chez un animal hôte : Telle souche de Proteus uréolytique in vitro, ne l'est plus in vivo (Ducluzeau et al., 1966). Telle souche de Lactobacillus, antagoniste puissant d'une souche de Streptococcus, coexiste très bien avec cette dernière in vivo (Ducluzeau et Raibaud, 1968). Inversement, un mélange de trois souches de Clostridium capable d'éliminer totalement in vivo une souche de Clostridium perfringens, est sans effet sur cette dernière dans un milieu de culture. Une autre souche de Clostridium, incapable de s'établir dans le tube digestif de souris axéniques recevant un certain régime alimentaire, croît cependant in vitro dans le contenu cæcal de ces mêmes animaux. Il est donc indispensable que le rôle métabolique des bactéries appartenant à une flore complexe soit étudié chez le gnotoxénique.

Pour identifier les bactéries actives dans une flore d'holoxénique, on a souvent intérêt à procéder par étape : la flore de l'holoxénique sera d'abord fractionnée par des traitements divers (dilutions, chauffages à diverses températures, acidification). Les diverses fractions seront associées à des animaux axéniques, puis on tentera d'isoler des fractions actives, sur une gamme de milieux de culture, le maximum de souches bactériennes pures appartenant à des espèces différentes. Ces souches seront alors administrées ensemble, puis si l'ensemble est actif, on cherchera le plus petit nombre de souches nécessaire et suffisant pour reproduire chez l'animal gnotoxénique l'activité métabolique recherchée. En procédant ainsi, Ducluzeau et al. (1977) ont réussi à isoler dans les fèces de souris holoxéniques deux souches de Clostridium qui, associées 
l'une ou l'autre à une souche d'Escherichia coli, ont un effet antagoniste vis-à-vis de Shigella flexneri chez des souris gnotoxéniques. De même, Sacquet et al. (1979) ont isolé du tube digestif de rats holoxéniques une souche de Clostridium capable de transformer l'acide $\beta$-muricholique en acide $\omega$-muricholique chez le rat gnotoxénique en pratiquant de tels fractionnements et en utilisant de surcroît des animaux holoxéniques porteurs d'une fistule du canal cholédoque, intervention qui a eu pour effel de réduire le nombre de souches bactériennes actives sur les sels biliaires.

Une étude de ce genre s'avère souvent très longue et peut nécessiter l'utilisation d'un grand nombre de modèles gnotoxéniques : plus de 1000 souris ont été nécessaires à l'isolement des deux souches de Clostridium actives sur S. flexneri en association avec E. coli (Ducluzeau et al., 1977).

Lorsque le rôle d'une souche ou d'une association de souches aura été démontré in vivo, dans le tube digestif d'animaux gnotoxéniques, alors seulement on cherchera à reproduire le phénomène in vitro pour en étudier le mécanisme. Après avoir établi qu'un ensemble de souches de Clostridium produisait de l'acide $\omega$-muricholique chez les rats gnotoxéniques, Sacquet et al. (1979) ont recherché la présence d'une 6-isomérase in vitro chez ces souches. La souche $\mathrm{R} 6 \times 76$, qui possède un tel système enzymatique, est capable de fabriquer l'acide $\omega$-muricholique lorsqu'on l'établit chez un rat gnotoxénique. La démarche qui aurait consisté à rechercher d'abord in vitro une 6-isomérase de l'acide $\beta$-muricholique sur des souches isolées dans divers milieux sélectifs chez un holoxénique n'aurait pas abouti à l'isolement de cette souche $R 6 \times 76$, parce qu'elle ne se développe pas sur les milieux sélectifs usuels. Seul, un fractionnement de la flore a permis d'éliminer la plupart des souches bactériennes inactives, tout en conservant la souche active.

Lorsque Hudault et al., au Laboratoire d'Ecologie microbienne, ont isolé une association de 3 souches capables d'éliminer $C$. perfringens du tube digestif de souris gnotoxéniques, ces auteurs ont essayé de reproduire le phénomène in vitro. Lorsqu'un faible inoculum de la souche cible de $C$. perfringens est introduit dans une suspension de fèces de ces souris gnotoxéniques, le développement de $C$. perfringens est inhibé. Donc, on peut reproduire in vitro le phénomène observé in vivo. Ces auteurs ont alors comparé le pouvoir inhibiteur du surnageant et celui du culot de centrifugation de la suspension fécale active. Ils ont constaté que seul le culot était actif. Cette observation leur a permis de rejeter un cerlain nombre d'hypothèses classiques sur l'effet de barrière microbien, hypothèses qui avaient été formulées à partir d'études effectuées sur des souches cultivées in vitro. Donc l'utilisation d'un modèle expérimental analytique permet d'orienter l'étude du mécanisme vers des hypothèses qu'une éfude in vitro n'aurait pas permis d'envisager.

L'utilisation de modèles gnotoxéniques de fype analytique est également indispensable si l'on veut connaître l'importance respective des enzymes de l'hôte et des enzymes de la flore dans les processus digestifs. Ducluzeau et al. (1966) et Moreau, Ducluzeau ef Raibaud (1976) ont montré qu'il n'existait pas d'uréase tissulaire chez le rat axénique ef que l'uréolyse était due à différentes souches bactériennes parmi lesquelles certaines espèces de Lactobacillus jouent un rôle important. Dans le cas de l'amylase chez le poulet, Szylit et al. (1978) ont constaté que l'activité amylolytique de certains Lactobacillus du jabot venait compléter l'action des amylases endogènes. Cette action synergique 
est nécessaire pour obtenir une dégradation maximale des grains d'amidon ingérés par l'animal gnotoxénique (Szylit et al., 1978). Chez le rat gnotoxénique, monoassocié à une souche de Clostridium et privé de ses sécrétions pancréatiques par ligature du canal pancréatique, Corring et al. ont montré que l'activité protéolytique de la population bactérienne s'avère suffisante pour compenser la perte des enzymes protéolytiques de l'hôte (Corring, Moreau et Ducluzeau, 1979).

2. - Variations de l'expression d'un effet biologique potentiel en fonction de l'environnement.

Le modèle expérimental analytique permet de mettre en évidence un effet biologique potentiel de souches bactériennes connues. L'application pratique des résultats obtenus sur de tels modèles analytiques passe évidemment par l'étude de leur expression en fonction de l'environnement. Il est donc indispensable de savoir si tel effet potentiel d'une souche ou d'une association de souches s'exprime, ou non, lorsque l'environnement biotique (microbes associés) ou abiotique (régime alimentaire, conditions climatiques) varie. Là encore l'animal gnotoxénique est indispensable pour étudier l'expression d'un effet biologique potentiel dans diverses situations écologiques.

Une des questions les plus souvent posées aux spécialistes de la flore du tube digestif par les nutritionnistes est de connaître l'impact d'un composant d'un régime alimentaire sur l'équilibre de la flore. Jusqu'à maintenant et, bien souvent encore, pour répondre à cette question, on entreprend de comparer les flores, en général fécales, de deux lots d'animaux, l'un recevant le composant étudié, l'autre non. Sur chaque échantillon, on peut réaliser une analyse différentielle quantitative de la flore sur les milieux de culture sélectifs dont on dispose. Compte tenu de la complexité de la flore d'un holoxénique, il faut considérer que, pour un laboratoire bien équipé, une telle analyse demande une demi-journée d'ensemencement ef environ une semaine de lecture. Le nombre des échantillons analysés sera forcément très réduit et dans la majeure partie des cas les variations observées entre 2 prélèvements successifs d'un même animal seront aussi importantes que les variations entre prélèvements provenant d'animaux de chaque lot. De plus, on saił qu'une fraction de la flore des holoxéniques échappera de toute façon à ces investigations, celle que l'on ne sait pas cultiver. Ainsi, l'équipe américaine de Finegold et Sutter (1978) a comparé les flores fécales d'hommes holoxéniques recevant 2 types de régimes alimentaires. Ce travail, qui a requis un effort technique et financier énorme, n'a cependant amené à aucune conclusion définitive concernant l'impact des régimes sur la flore.

On peut également dans ce type de recherches limiter l'analyse à quelques espèces bactériennes faciles à dénombrer sélectivement comme $E$. coli ou Lactobacillus. Dans ce cas, les prélèvements analysés peuvent être bien plus nombreux, mais l'interprétation reste difficile. La constance du nombre de bactéries appartenant à une population sousdominante peut être interprétée comme liée à la persistance des associations de bactéries de la flore dominante qui répriment ladite population sous-dominante et donc être un argument en faveur de l'absence d'action du composant alimentaire considéré sur la flore de l'hôte. Mais bien entendu, le composant alimentaire peut agir sur d'autres bactéries que celles dénombrées sans que l'on ait les moyens de s'en apercevoir. Au contraire, l'utilisation de modèles analytiques apporte des réponses peut-être limitées, 
mais toujours claires. On peut par exemple savoir si le régime alimentaire esł capable d'agir sur l'établissement d'une souche déterminée de bactéries.

Des modèles analytiques constitués par des souris axéniques monoassociées à un Clostridium nous ont permis de démontrer un effet direct du régime alimentaire sur l'établissement de ce Clostridium. En effet, celui-ci ne s'établit pas en présence de certains régimes commerciaux pour rongeurs, mais s'établit lorsque ces animaux reçoivent des laits d'origine animale très variée ou des laits de remplacement. Nous avons montré que les facteurs qui agissent sur l'implantation de la souche ne se trouvaient pas dans la fraction lipidique de l'aliment. L'utilisation de ces modèles monoxéniques nous ont apporté un résultat plus inattendu : si la souche de Clostridium est établie dans le tube digestif de souris ingérant un des régimes lactés convenables, dit permissif, elle reste présente à niveau élevé lorsque le régime permissif est remplacé par un des régimes commerciaux qui ne permet pas l'établissement initial du Clostridium. Ce résultat peut être de grande importance pour comprendre l'effet du régime sur la séquence d'établissement des bactéries chez le jeune animal. Certes, on peut objecter que de tels résultats sont ponctuels car ils ne portent que sur quelques-unes des innombrables bactéries de la flore des holoxéniques et qu'ils sont obtenus sur des modèles artificiels dont la flore ne ressemble en rien à celle d'un holoxénique. Ils permettent toutefois d'affirmer un rôle du régime sur certains éléments de la flore. L'effet de rémanence a été maintenant observé pour d'autres bactéries que le Clostridium cité plus haut, on peut donc penser qu'il a une certaine généralité. De plus, on peut maintenant envisager des modèles plus complexes, où le Clostridium peut être marqué pour être reconnu spécifiquement et associé ensuite à d'autres bactéries. On sera alors à même d'étudier le phénomène dans d'autres conditions plus proches de l'holoxénique. Ainsi, en associant une souche de microcoque à la souche de Clostridium, on a vu s'établir ces deux souches avec un régime commercial qui ne permet pas l'implantation du Clostridium seul. Dans cet exemple d'ailleurs, le microcoque a été réprimé par le Clostridium au bout d'une semaine, l'effet de rémanence se substituant alors à l'effet adjuvant du microcoque.

L'utilisation de modèles gnotoxéniques a permis d'établir que le régime alimentaire pouvait agir de façon indirecte sur le développement d'une souche bactérienne dans le tube digestif, en modifiant l'expression de l'effet de barrière microbien. Nicolas et al., au Laboratoire d'Ecologie Microbienne, ont montré que trois souches de bactéries ancérobies exercent un effet de barrière drastique sur $C$. perfringens chez les souris gnotoxéniques, à condition que le régime alimentaire soit stérilisé par irradiation. Si le même régime alimentaire est autoclavé, l'effet de barrière devient seulement permissif, les souris deviennent des « porteurs sains » de $C$. perfringens. Un autre exemple montrera que seuls des modèles gnotoxéniques peuvent apporter des solutions à certains problèmes biologiques. Lorsqu'on étudie les lactobacilles dans le tube digestif de souriceaux holoxéniques allaités, on peut voir qu'une ou deux espèces dominent, alors que d'autres sont peu fréquentes et rarement présentes dans la flore dominante. Deux hypothèses sont plausibles : soit le lait de la mère ne permet pas l'établissement de l'espèce sous-dominante dans le tube digestif de ses souriceaux : dans ce cas, le régime alimentaire réprimerait la capacité du lactobacille à coloniser le tube digestif d'un nouveau-né ; soit les autres espèces de lactobacilles exercent un effet de barrière sur l'espèce dominante : il s'agirait alors d'un antagonisme bactérien qui s'oppose à 
l'expression de la capacité à coloniser le tube digestif d'une bactérie. Nous avons comparé la cinétique de l'implantation dans le tube digestif de souriceaux monoxéniques, entre la naissance et le sevrage, d'une souche de lactobacilles appartenant à l'espèce sous-dominante et celle d'une souche de lactobacilles appartenant à l'espèce dominante. Nous avons constaté que la première souche s'établissait moins vite et à un niveau moins élevé que la deuxième ; dans ce cas la réponse est claire : la nature du régime alimentaire suffit à expliquer les différences dans le niveau d'implantation des deux espèces de lactobacilles chez l'holoxénique.

Un exemple frappant où l'effet potentiel d'une souche dépend étroitement des conditions écologiques est l'activité antagoniste de Bacillus licheniformis vis-à-vis de C. perfringens (Ducluzeau et al., 1976 ; Ducluzeau et al., 1978a). B. licheniformis produit in vivo un antibiotique ressemblant à la bacitracine et de ce fait empêche radicalement l'établissement ultérieur de $C$. perfringens. Mais il suffit d'associer une souche de lactobacille, présente chez tous les holoxéniques, à $B$. licheniformis pour que l'effet antagoniste de cette dernière souche vis-à-vis de $C$. perfringens disparaisse totalement. L'élimination passive de souches cibles variées dans le tube digestif de souris holoxénique (Ducluzeau, Bellier et Raibaud, 1970), alors que ces souches cibles se développent dans le tube digestif de souris axéniques, est due à l'effet de barrière microbien. Pour que l'on puisse utiliser dans la pratique cet effet de barrière pour protéger le tube digestif d'un animal contre l'invasion de bactéries potentiellement pathogènes, il est nécessaire de déterminer la ou les associations microbiennes qui répriment la capacité à coloniser de ces bactéries en conditions holoxéniques. Il faut donc, à l'aide de modèles gnotoxéniques, étudier l'expression de l'effet de barrière en faisant varier l'environnement microbien. Nicolas et al., au Laboratoire d'Ecologie microbienne, ont montré que l'effet de barrière drastique contre $C$. perfringens, dû à une association de trois souches, s'exprimait de la même façon quand on associait à ces 3 souches 33 autres souches isolées de la flore dominante d'holoxéniques, ce qui n'était pas le cas lorsqu'on utilisait comme souche antagoniste $B$. licheniformis.

L'expression d'un effet métabolique potentiel d'une souche donnée peut aussi varier en fonction des conditions d'environnement. Souvent, la capacité d'une souche anaérobie stricte à coloniser le fube digestif d'un axénique ne s'exprime que chez un monoxénique hébergeant une souche qui abaisse le potentiel d'oxydoréduction du contenu digestif de l'axénique à un niveau compatible avec le démarrage de la souche anaérobie stricte. Gustafsson, Midvedt et Norman (1968) ont montré que l'activité $7 \alpha$ déhydroxylase s'exprimait dans le tube digestif d'un gnotoxénique à la condition qu'une souche déconjugant les sels biliaires in vivo lui soit associée. II en est de même pour la souche possédant une 6-épimérase de l'acide $\beta$-muricholique (Sacquet et al., 1979).

Un autre exemple permettra de montrer comment l'utilisation de modèles gnotoxéniques permet de mieux cerner la réalité de phénomènes complexes qu'une observation globale chez des holoxéniques faisait considérer comme simples. II était admis jusqu'ici que l'addition de faibles doses d'antibiotiques dans l'alimentation entraînait la sélection dans la flore de bactéries résistantes à cet antibiotique. En fait, la gnotoxénie a montré que l'effet initial de ces antibiotiques était d'éliminer les barrières microbiologiques exercées par la flore dominante de l'hôte à l'égard des bactéries antibio- 
résistantes de l'environnement. Les bactéries de barrière, souvent anaérobies strictes, sont en effet très sensibles aux antibiotiques. Ainsi, Tancrède et al. (1977) ont observé, dans le cas d'un modèle gnotoxénique comportant une souche d'E. coli réprimée par une flore de barrière complexe, que, après ingestion d'ampicilline, la souche d'E. coli se multipliait quelques heures après le début du traitement dans la flore de ces souris alors qu'elle était toujours sensible à l'antibiotique ingéré. Pourtant, Duval et al., au Laboratoire d'Ecologie microbienne, ont montré que cette même dose d'antibiotique élimine complètement la population de la même souche d'E. coli monoassociée à une souris axénique. L'apparition de mulants résistant à l'ampicilline est plus tardive chez la souris hébergeant une flore complexe comme chez la souris monoxénique et des clones sensibles coexistent alors avec les clones résistants.

Un dernier exemple illustre l'intérêt du modèle expérimental analytique en pathologie du tube digestif. Pour démontrer l'étiologie infectieuse d'une maladie, le postulat de Koch-Henlé exige d'isoler chez tous les individus malades un agent infectieux avec lequel on puisse reproduire la maladie chez des individus sains. Dans le cas d'une maladie infectieuse à point de départ digestive, l'isolement de l'agent infectieux bactérien est souvent une étape difficile à franchir à cause de la complexité de la flore du tube digestif, mais surtout la reproduction de la maladie par ingestion de l'agent infectieux s'avère impossible chez les holoxéniques sains. En effet, les animaux sains possèdent des barrières microbiologiques très efficaces à l'égard des pathogènes de l'environnement. Le développement d'un agent infectieux ne peut donc avoir lieu qu'à la faveur de la rupture accidentelle des barrières microbiologiques et cette rupture est bien difficile à obtenir expérimentalement lorsqu'on ne connaît pas les bactéries responsables de l'effet de barrière. Inversement, chez l'axénique, les barrières microbiologiques n'existent pas et on peut facilement mettre en évidence le pouvoir pathogène potentiel de tout agent microbien. Dabard ef al. (1979) ont ainsi démontré le rôle de Clostridium difficile dans l'étiologie de la diarrhée néonatale mortelle du levraut. Ils ont constaté de plus que cette bactérie exerçait son pouvoir pathogène non pas seule, mais en synergie avec d'autres Clostridium comme $C$. perfringens. Chez l'homme, le rôle pathogène de $C$. difficile est évoqué depuis quelques temps dans l'étiologie de la dangereuse colite ulcéromembraneuse (Bartlett et al., 1978). Mais la reproduction de la maladie chez le hamster holoxénique n'avait jusqu'ici été réussie qu'après un traitement antibiotique des hamsters suivi d'une inoculation per os de C. difficile (Lusk ef al., 1978). Dans le travail de Dabard ef al., 1979, non seulement la maladie a été reproduite chez le levraut gnotoxénique sans traitement antibiotique préalable, mais le fait d'une synergie entre 2 souches de Clostridium pouvant être nécessaire à l'expression du pouvoir pathogène de l'un d'eux a été démontré pour la première fois.

\section{3. - L’animal gnotoxénique, modèle expérimental mimétique.}

Conditions ef limites d'utilisation des modèles mimétiques.

Lorsque l'on cherche à utiliser un animal gnotoxénique comme modèle de type mimétique, on a généralement recours à la souris ou parfois au rat axéniques, ensemencés avec la totalité ou des fractions de la flore d'animaux ou d'hommes holoxéniques. La valeur du modèle ainsi obtenu dépend naturellement des possibilités d'éta- 
blissement des souches de provenance diverse ensemencées chez la souris, et de l'homologie entre les équilibres bactériens obtenus chez la souris modèle et chez l'animal d'origine. On constate d'abord que la plupart des souches bactériennes, quel que soit l'animal dont elles proviennent, sont capables de s'établir, c'est-à-dire de se multiplier et de subsister indéfiniment en nombre élevé dans le fube digestif de la souris ou du rat gnotoxénique. Les quelques exceptions à cette règle s'expliquent en général par des exigences spéciales des bactéries (souches psychrophiles ou thermophiles strictes).

Pour apprécier le degré d'homologie que l'on peut atteindre en transférant une flore complexe d'un animal holoxénique à la souris axénique, Ducluzeau et al. (1978b) ont réalisé l'expérience suivante. La flore fécale de truies et de porcelets holoxéniques a été administrée per os chez des souris axéniques. Les souris ainsi ensemencées ont été maintenues en isolateurs pendant plusieurs mois, puis la flore fécale des souris ayant reçu la flore de porcelet a été ensemencée chez un porcelet axénique. Un certain nombre de groupes microbiens ont été dénombrés dans les selles des différents animaux à l'aide de techniques d'analyse différentielle quantitative. Ces auteurs ont observé que le modèle souris gnotoxénique reproduit plus fidèlement la flore du porcelet que celle du porc adulte. Dans ce dernier cas toutefois, la souris gnotoxénique représente le meilleur moyen pour conserver l'ensemble des groupes microbiens dénombrés qui proviennent du porc, surtout si le prélèvement de la flore fécale a lieu en anaérobiose stricte. Dans le cas où une même flore provenant d'un porcelet holoxénique est ensemencée simultanément chez une souris et un porcelet gnotoxéniques, placés dans un même isolateur dans des conditions de régime alimentaire et d'environnement identiques, les résultats observés chez la souris modèle peuvent être extrapolés au porcelet. Cela n'est toutefois valable que pour les expériences de courte durée. Si l'association entre la flore du porcelet et la souris hôte se prolonge, des modifications apparaissent dans le faciès microbien, mais elles semblent réversibles, lorsque cette flore est transférée à un nouveau receveur axénique, porcelet ou souris.

La réponse que l'on peut donner à la question de savoir si la souris gnotoxénique représente un bon modèle pour étudier la flore du porc holoxénique n'est donc pas univoque ; la valeur de ce modèle dépend de la nature de la flore de l'animal donneur, de la durée d'association entre la flore ef la souris hôte et dans une certaine mesure, de la nature du régime alimentaire. Ce modèle de type mimétique reste malgré ses imperfections le plus utile que nous possédions actuellement dans tous les cas où l'on ne peut pas expérimenter directement sur l'animal concerné.

Il faut aussi avoir présent à l'esprit qu'un modèle mimétique donne une image "figée » de la flore de l'animal donneur. En effet la souris modèle est maintenue en isolateur, donc à l'abri des bactéries de l'environnement, alors que ces dernières entrent en permanence en contact avec l'holoxénique. Nous avons montré au Laboratoire d'Ecologie microbienne que le maintien, en isolateur, de souris à flore complexe n'entraîne ni une simplification de la flore par disparition de nombreuses espèces bactériennes comme on l'a cru un moment, ni une disparition totale des fluctuations de la flore sous-dominante. II n'en reste pas moins que ces conditions d'isolement strict constituent une autre différence entre l'animal modèle et l'animal donneur.

II ne faudrait toutefois pas croire que l'on peut supprimer cette différence en faisant vivre les souris gnotoxéniques modèles, ensemencées avec la flore des holo- 
xéniques, dans le même environnement que les holoxéniques. Dans ces conditions leur flore évolverait rapidement vers celles de souris holoxéniques ; c'est ce que l'on observe par exemple chez certaines souris SPF qui, bien qu'isolées de l'environnement par une barrière mécanique sévère, mais non hermétique, se révèlent porteuses de bactéries typiques des souris holoxéniques qui ne leur ont jamais été inoculées. Cet exemple montre donc qu'il existe des bactéries qui, pour des raisons encore inconnues, sont mieux « adaptées » que d'autres à une espèce animale donnée. Un animal gnotoxénique ne restera un modèle mimétique qu'autant qu'il sera élevé dans des conditions d'isolement microbiologique strictes que seules les techniques de l'axénie peuvent lui assurer.

Si l'on cherche à l'aide d'un modèle de type mimétique à étudier non seulement les équilibres microbiens, mais encore l'impact de la flore sur la physiologie de l'hôte, de grandes précautions doivent être prises. Ainsi les conséquences d'une décontamination du tube digestif de l'homme ne peuvent être prévues en utilisant un modèle mimétique tel que rat ou souris axéniques hébergeant une flore humaine. En effet, le rongeur axénique développe un cæcum de 5 à 6 fois plus volumineux que celui du rongeur holoxénique ou associé à une flore humaine. Bien des caractéristiques du rat axénique disparaissent lorsque l'on fait l'ablation de son cæcum et sont donc liées à la distension cæcale et non à l'effet de la flore (Wostmann, Bruckner-Kardoss et Knight, 1968). Mais la distension cæcale n'est pas une caractéristique liée seulement à l'état d'axénie, elle est liée aussi à l'hôte axénique. Le porcelet, le poulet et probablement l'enfant axéniques ne présentent pas cette distension cæcale. Par contre, d'autres caractéristiques telles que l'amincissement de la paroi digestive et la pauvreté de la muqueuse du tube digestif en cellules immunocompétentes semblent être des caractéristiques très générales des axéniques. En fonction du phénomène biologique éłudié, on pourra donc être amené à choisir comme modèle mimétique pour l'homme le porcelet axénique plutôt que le rongeur axénique.

L'utilisation de modèles mimétiques expérimentaux est très récente. Ceci explique que les données de la littérature soienf peu nombreuses et ne permettent pas de porter un jugement définitif sur l'intérêt de ces modèles. Cependant, Corpet et Nicolas (1979) ont récemment montré qu'il était possible d'isoler, à partir de souris axéniques ensemencées avec la flore fécale d'un porcelet holoxénique, 7 souches bactériennes qui exercent un effet de barrière drastique sur $C$. perfringens à la fois chez la souris gnotoxénique et chez le porcelet gnotoxénique. Ceci confirme les données de Ducluzeau et al. qui montrent que le tube digestif de la souris axénique est un biolope favorable au développement de souches bactériennes d'origine porcine... On est donc en droit de penser que ces 7 souches jouent un rôle dans la protection du porcelet holoxénique visà-vis de $C$. perfringens. L'étape ultérieure qui reste à franchir est de contrôler chez le porcelet holoxénique l'établissement de ces souches et de le mettre en rapport avec la présence ou l'absence de $C$. perfringens. L'avantage du modèle mimétique est ici évident, si l'on pense aux difficultés techniques et au coût qu'aurait représenté le tri de ces 7 souches en utilisant seulement le porcelet axénique.

En ce qui concerne l'application du modèle mimétique à l'étude de la flore humaine, les résultats de Tancrède et al. (1977) sont encourageants. Ces auteurs ont réussi à transférer chez des souris axéniques une population bactérienne complexe issue de 
fèces humaines qui exerce un effet de barrière permissif vis-à-vis de $E$. coli. Le niveau d'établissement de $E$. coli dans les fèces du donneur humain était sensiblement le même que celui que l'on observe chez les souris-homme une semaine après leur ensemencement. L'étape ultérieure qui consiste à isoler l'association bactérienne minimale ayant un effet de barrière permissif sur $E$. coli n'a pas été franchie, mais on constate une excellente stabilité dans le temps de l'effet de barrière anti $E$. coli chez les sourishomme et le transfert de cet effet à de nouvelles souris axéniques.

L'utilisation de souris axéniques, en tant que modèle mimétique expérimental, permet aussi d'aborder dans une large mesure l'étude des phénomènes biologiques qui dépendent à la fois des bactéries du tube digestif et du régime alimentaire de l'hôte, puisque la souris acceple des types de régime alimentaire très variés.

La comparaison entre le modèle mimétique et le modèle analytique - par exemple souris-porcelet ef porcelet - peut aussi mettre en évidence un effet insoupçonné de l'hôte s'il y a discordance entre les résultats obtenus avec les deux modèles. Hudault et al. ont ainsi observé qu'une barrière drastique contre $C$. perfringens chez la souris gnotoxénique n'était que permissive chez le rat gnotoxénique hébergeant les mêmes souches et recevant le même régime alimentaire.

En conclusion, le modèle mimétique expérimental, s'il est utilisé avec discernement, doit pouvoir rendre de grands services pour étudier le rôle de certaines bactéries qu'hébergent l'homme et les animaux d'intérêt zootechnique et il doit permettre de franchir un certain nombre d'étapes obligatoires dans le contrôle de la flore du tube digestif chez l'holoxénique.

Journées Ingestion-Digestion-Absorption de l'Association française de Nutrition, Paris, 15-16 novembre 1979.

\section{Références}

BARTLETT J. G., WEN CHANG T., GURWITH M., GORBACH S. L., ONDERDONK A. B., 1978. Antibiotic-associated pseudomembranous colitis due to toxin-producing clostridia. N. Engl. J. Med., 298, 531-534.

CORPET D., NICOLAS J. L., 1979. Antagonistic effect of intestinal bacteria from the microflora of holoxenic (conventional) piglets, against Clostridium perfringens in the digestive tract of gnotoxenic mice and gnotoxenic piglets. In FLIEDNER et al., Clin. experim. Gnotobiotics, Zbl. Bakt., Suppl. 7, 169-174.

CORRING T., MOREAU C., DUCLUZEAU R., 1979. Comparative apparent digestibility of casein in holoxenic, axenic, and Clostridium bifermentans monoassociated rats. Am. J. clin. Nufr., 32, $1231-1237$.

DABARD J., DUBOS F., MARTINET L., DUCLUZEAU R., 1979. Experimental reproduction of neonatal diarrhea in young gnotobiotic hares simultaneously associated with Clostridium difficile and other Clostridium strains. Infect. Immun., 24, 7-11.

DUCLUZEAU R., RAIBAUD P., DICKINSON A. B., SACQUET E., MOCQUOT G., 1966. Hydrolyse de l'urée in vifro el in vivo, dans le cæcum de rats gnotobiotiques, par différentes souches bactériennes isolées du fube digestif de rats conventionnels. C. R. Acad. Sci. Paris, Sér. D, 262, 944947.

DUCLUZEAU R., RAIBAUD P., 1968. Lyse in vitro de certains types de Lactobacillus par une souche de Streptococcus liquefaciens : Incidence du phénomène sur l'équilibre entre ces bactéries dans le tube digestif de souris "gnotoxéniques ». C. R. Acad. Sci. Paris, sér. D, 266, 1332-1334.

DUCLUZEAU R., BELLIER M., RAIBAUD P., 1970. Transit digestif de divers inoculums bactériens introduits per os chez des souris axéniques et « holoxéniques » (conventionnelles) : effet anta- 
goniste de la microflore du tractus gastro-intestinal. Zbl. Bakt. Parasit. Infectionskr. Hyg., 1 Orig., 213, 533-548.

DUCLUZEAU R., RAIBAUD P., LAUVERGEON B., GOUET Ph., RIOU Y., GRISCELLI C., GHNASSIA J. C., 1975. Immediate postnatal decontamination as a means of obtaining axenic animals and human infants. Can. J. Microbiol., 4, 563-566.

DUCLUZEAU R., DUBOS F., RAIBAUD P., ABRAMS G. D., 1976. Inhibition of Clostridium perfringens by an antibiotic substance produced by Bocillus licheniformis in the digestive tract of gnotobiotic mice : effect on other bacteria from the digestive tract. Antimicrob. Agents Chemother., 9 , 20-25.

DUCLUZEAU R., LADIRE M., CALLUT C., RAIBAUD P., ABRAMS G. D., 1977. Antagonistic effect of extremely oxygen-sensitive Clostridia from the microflora of conventional mice and of Escherichia coli against Shigella flexneri in the digestive tract of gnotobiotic mice. Infect. Immun., 17, 415-424.

DUCLUZEAU R., DUBOS F., RAIBAUD P., ABRAMS G. D., 1978a. Production of an antibiotic substance by Bacillus licheniformis within the digestive tract of gnotobiotic mice. Antimicrob. Agents Chemother., 13, 97-103.

DUCLUZEAU R., RAPINE Ph., COURVALIN C., RAIBAUD P., 1978b. Transfert de la flore microbienne fécale de porcelets et de porcs adultes holoxéniques à des souris adultes et des porcelets axéniques : effet de l'animal hôte et du régime alimentaire sur le faciès microbien du tube digestif des divers animaux. Ann. Microbiol. (Inst. Pasieur), 129B, 597-612.

DUCLUZEAU R., MOREAU C., CORPET D., TANCREDE C., MEYER D., SAINT-MARTIN M., 1969. Improvement of techniques leading to time sparing in rearing gnotoxenic animals. In FLIEDNER et al., Clin. experim. Gnotobiotics, Zbl. Bakt., Suppl. 7, 83-85.

FINEGOLD S. M., SUTTER V. L., 1978. Fecal flora in different populations, with special reference to diet. Am. J. clin. Nutr., S 116-S 122.

GUSTAFSSON B. E., MIDVEDT T., NORMAN A., 1968. Metabolism of cholic acid in germ-free animals after the establishment in the intestinal tract of deconjugating and $7 \alpha$-dehydroxylating bacteria. Acta pathol. microbiol. scand., 72, 433-443.

LUSK R. H., FETEKY R., SILVA J., BROWNE R. A., RINGLER D. H., ABRAMS G. D., 1978. Clindamycin-induced enterocolitis in hamsters. J. infect. Dis., 137, 464-475.

MOREAU C., DUCLUZEAU R., RAIBAUD P., 1976. Hydrolysis of urea in the gastro-intestinal tract of « monoxenic » rats : effect of immunization with strains of ureolytic bacteria. Infect. Immun., 13, 9-15.

MOREAU C., DUCLUZEAU R., MEYER D., 1978. Utilisation d'isolateurs miniaturisés pour l'expérimentation sur animaux gnotoxéniques. Sci. Tech. Anim. Lab., 3, 117-120.

RAIBAUD P., DUCLUZEAU R., MULLER M.-C., GHNASSIA J.-C., VERON M., GRISCELLI C., 1975. Evolution de la flore bactérienne fécale d'un enfant « hétéroxénique » élevé dans un isolateur et essai de décontamination par antibiothérapie. Ann. Microbiol. (Inst. Pasteur), 126B, 357-366.

SACQUET E., RAIBAUD P. M., MEJEAN C., RIOTTOT M. J., LEPRINCE C., LÉGLISE P. C., 1979. Bacterial formation of $\omega$-muricholic acid in rats. Appl. environ. Microbiol., 37, 1127-1131.

SAINT-MARTIN B., DUCLUZEAU R., GHNASSIA J.-C., GRISCELLI C., LAUVERGEON B., 1975. Amélioration de la technologie des enceintes stériles permettant leur application à la médecine et à la chirurgie. Rev. franç. Gynéc., 70, 585-593.

SZYLIT O., CHARLET G., CHAMPM., POPOT F., LE COZY., GALPIN J., RAIBAUDP., 1978 . Influence of the gnotoxenic state on the utilization of carbohydrates by chicken. VIth int. Symp. Gnotobiology, Ulm, 23 (Abstr.).

TANCREDE C., AZIZI P., RAIBAUD P., DUCLUZEAU R., 1977. Conséquences de la destruction des barrières écologiques de la flore du tube digestif par les antibiotiques. Perturbations des relations entre l'hôte et les bactéries potentiellement pathogènes. Méd. Mal. infect., 7, 145-149.

WOSTMANN B. S., BRUCKNER-KARDOSS E., KNIGHT P. L. Jr., 1968. Cecal enlargement, cardiac output and oxygen consumption in germfree rats. Proc. Soc. exp. Biol. Med., 128, 137-141. 